\title{
Aerodynamic moment characteristics of tandem-scheme aircraft
}

\author{
Illia Kryvokhatko \\ Antonov Company, Aerodynamics and Flight Dynamics Department, 03062 Akademika Tupoleva \\ St., 1, Kyiv, Ukraine
}

\begin{abstract}
Aerodynamic interference between forward and back wings of tandem-scheme aircraft significantly affects its pitch and roll moments. The interference increases roll stability in a narrow range of sideslip angles; there is a kink on the dependence of roll moment coefficient versus sideslip angle (that is not observed for conventional-scheme aircraft). Directional stability is decreased by a dihedral angle of forward wings and winglets on them but is increased by the same factors for back wings. If back wings' bending is significant, then aerodynamic interference may affect directional stability as well. The vortex system of tandem-wings at a sideslip angle was modeled incorrectly by the used CFD method (solving RANS), and further research is needed. The analytical and experimental methods show a good agreement concerning moment characteristics.
\end{abstract}

\section{Introduction}

In the beginning of the XXI century aerodynamic scheme "tandem" (with comparable areas of forward wings and back wings) became widespread among UAVs and small piloted aircrafts (fig. 1) [1-4].

Its aerodynamics has specifics caused by inevitable wing-wing interference. The tip vortices of the forward wings affect the flow on the back wings, create upwash and downwash (fig. 2). For a small vertical gap, forward wings also slow down the flow on the back wings and make it turbulent, but this effect might be low for reasonable parameters of configuration. Nevertheless, downwash and upwash always affect total lift coefficient and moment characteristics that concern the stability of aircraft and is of high importance.

\section{Object and methods}

For aerodynamic moments determination the following methods were used:

1) The analytical method developed by the author $[5,6]$;

2) The CFD method (RANS solving with Ansys software) [7];

3) Experimental research (in certified wind tunnel AT-1 at Antonov Company fig. 3,4 ) was performed for determination of the aerodynamic characteristics of the tandem-scheme UAV model [8]. 


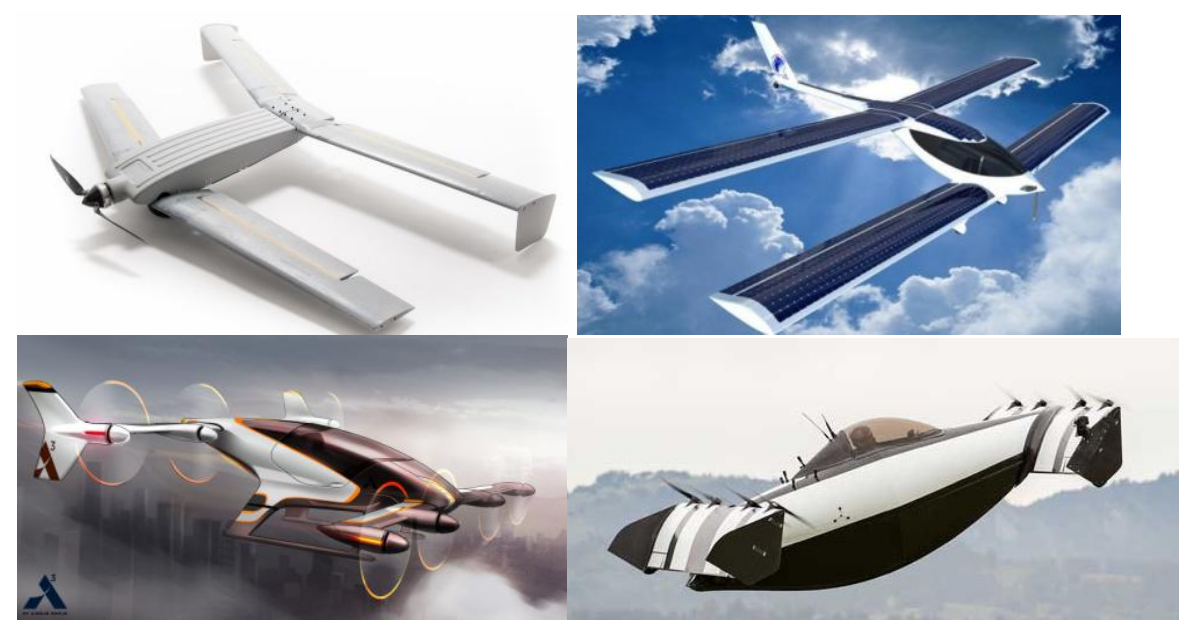

Fig. 1. «Vector Hawk» (Lockheed Martin, USA, 2014); «Eraole» (France, 2016); Design of air taxi «Vahana» (Airbus); «Opener BlackFly» (USA, 2014)

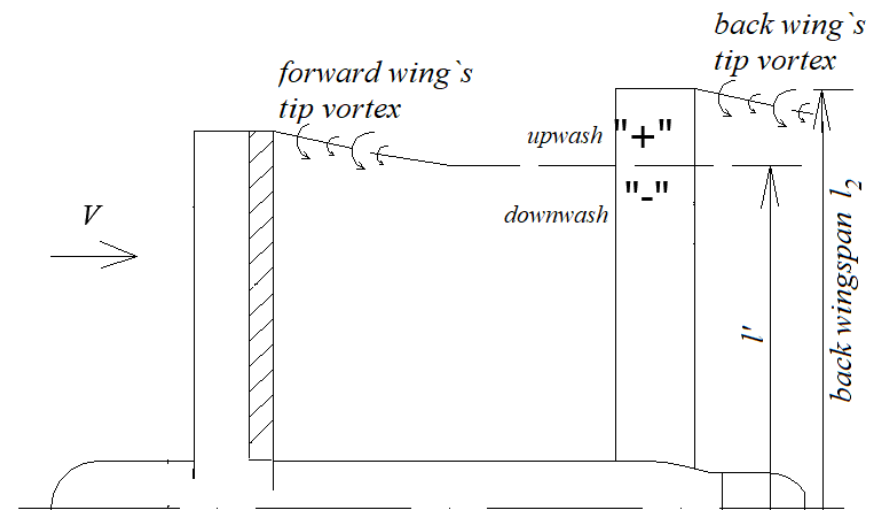

Fig. 2. Downwash/upwash flow pattern with the right forward wing`s tip vortex
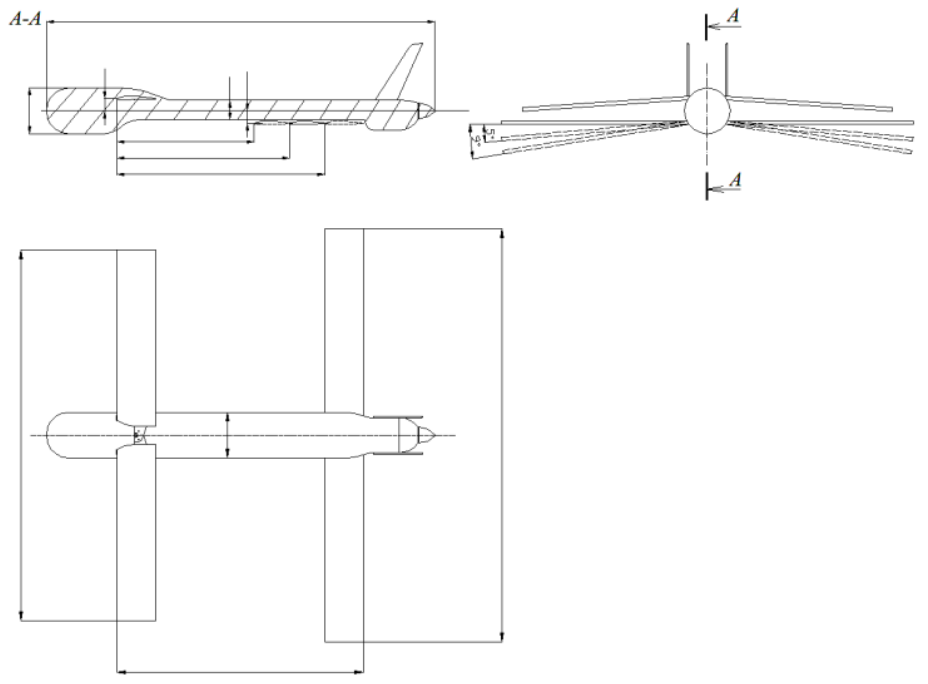

Fig. 3. Wind tunnel model of tandem-scheme UAV 


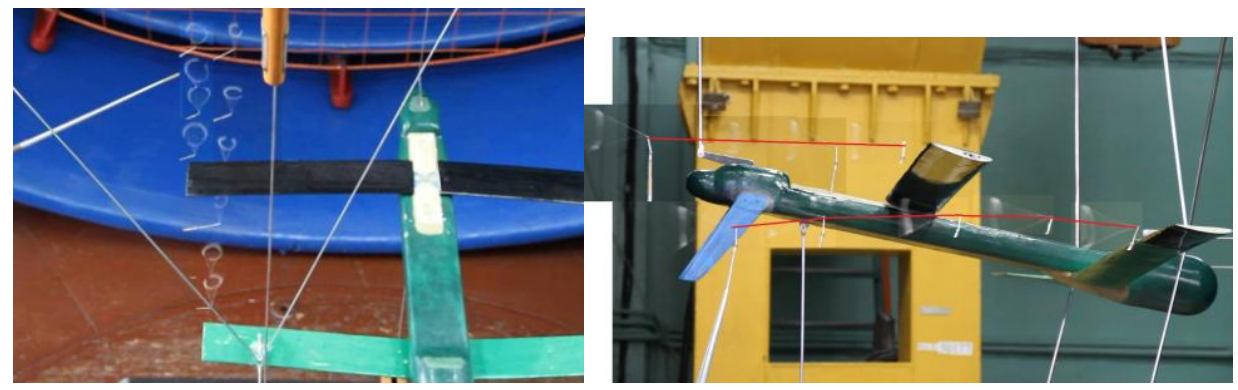

Fig. 4. Flow visualization over wind tunnel model of tandem-scheme UAV

\section{Pitch static moment}

Pitch static moment can be found only with the correct calculation of average downwash angle and therefore back wings` lift coefficient (fig. 5). The classic analytical method (by Prandtl) considers wingspan ratio, height, and stagger. The proposed method can take into account the wings ' dihedral angles and the sideslip angle as well. For simple cases, both methods show the same results. For geometries with dihedral angles, accurate pitch moment prediction by the new method is preferable to achieve minimal balancing losses for maximal lift-drag ratio at cruise angle of attack (fig. 6).

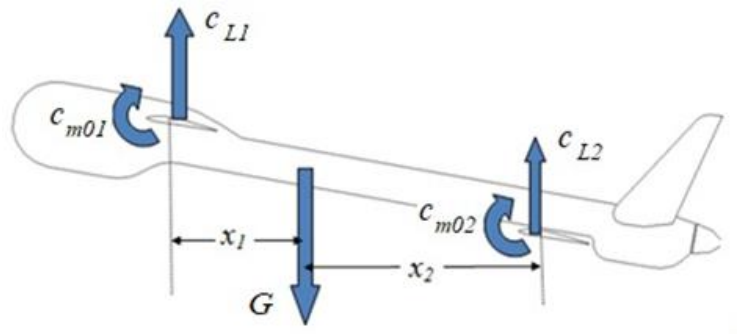

Fig. 5. Pitch moment scheme (moment of fuselage is considered as negligible)

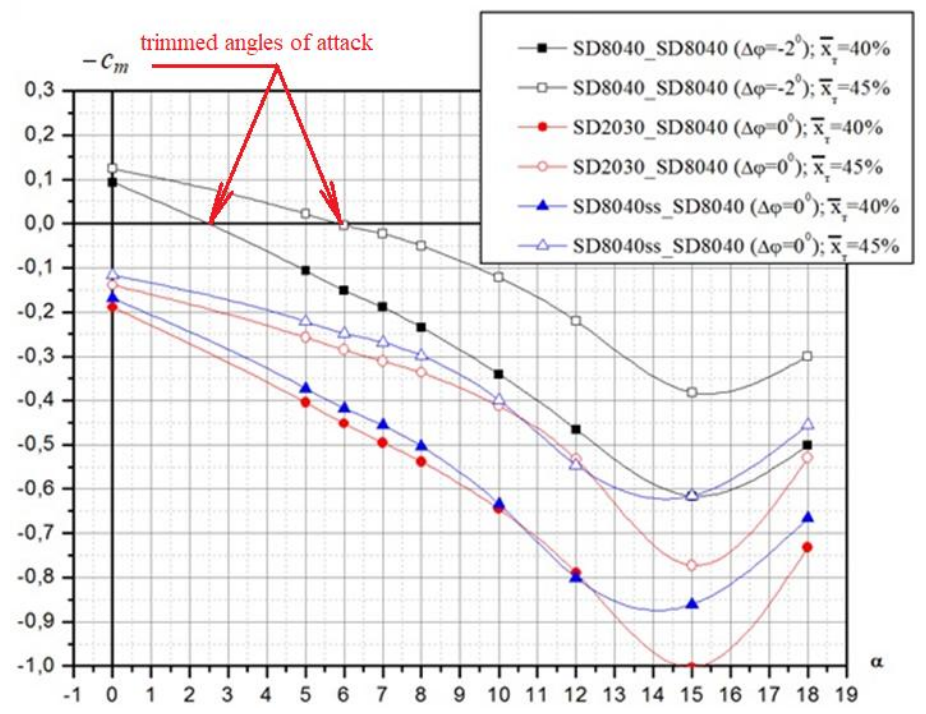

Fig. 6. Pitch moment coefficient dependences versus angle of attack (for different pairs of airfoils and different $C G$ positions) 


\section{Roll static moment}

The scheme of forces that create a roll moment for tandem aircraft is presented in fig. 7 .

Roll static moment dependence differs from the one of traditional aircraft. The interference of forward and back wings results in roll static stability increasing for low sideslip angles, but it rapidly decreases after $\beta=5 \ldots 10^{\circ}$ (fig. 8). This kink on the roll moment coefficient graph was predicted by the proposed analytical method and was observed in wind tunnel test data. Nevertheless, this effect was not detected by the CFD method based on RANS solving.

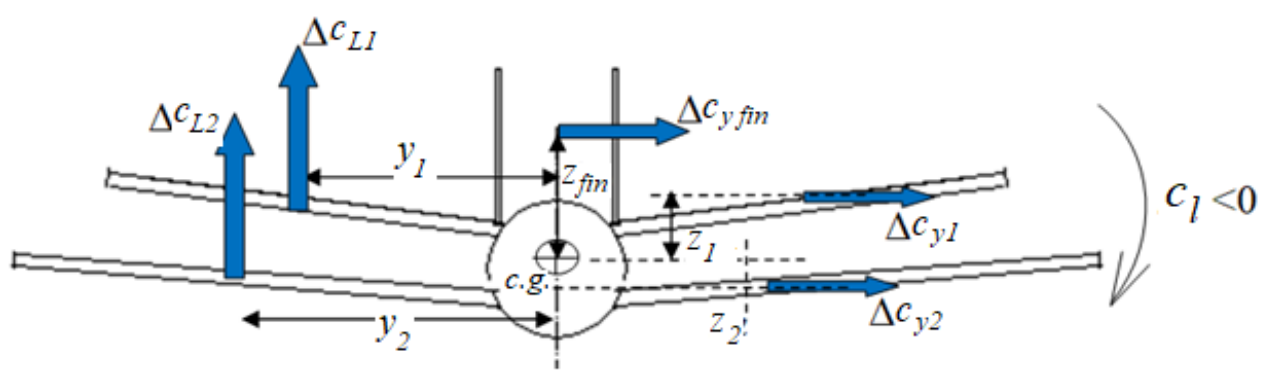

Fig. 7. Roll moment scheme

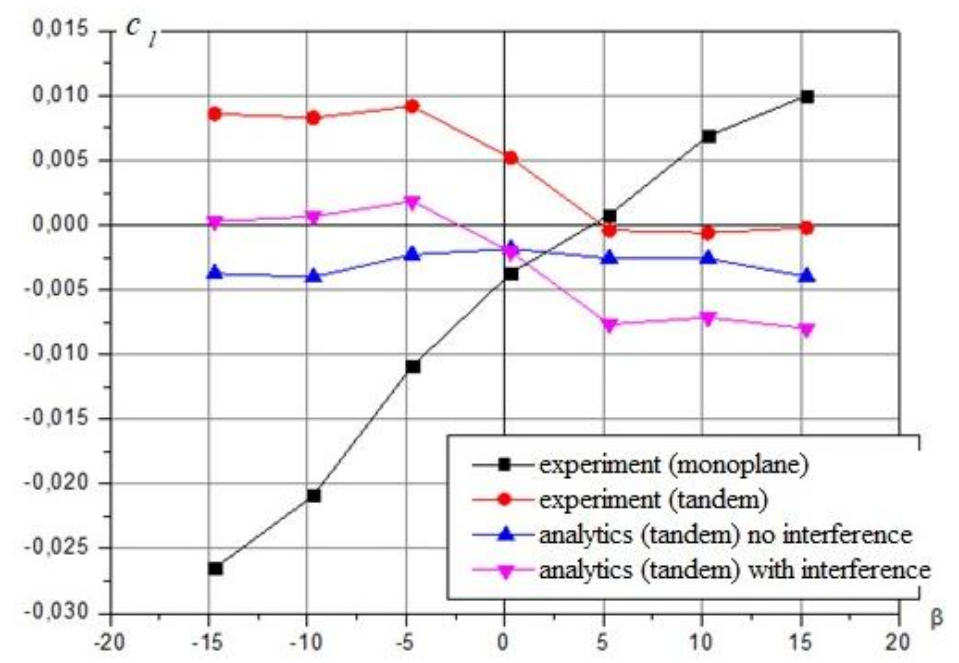

Fig. 8. Roll moment dependences

For an explanation of the effect, we should consider the position of forward wings 'tip vortices. Till both forward vortices are inside the span of back wings, the additional rolling stability is provided (fig. 9a). At some sideslip angle $\left(\beta=6 \ldots 8^{\circ}\right)$, the left forward tip vortex intersects the tip section of the back wing (fig. 9b). At higher sideslip angles, this particular vortex decreases rolling stability, while right forward tip vortex still increases. So after certain sideslip angle (we may call it kink $\beta$ ), the aerodynamic interference has almost no effect on rolling stability, and it can be estimated as for isolated forward and back wings. 

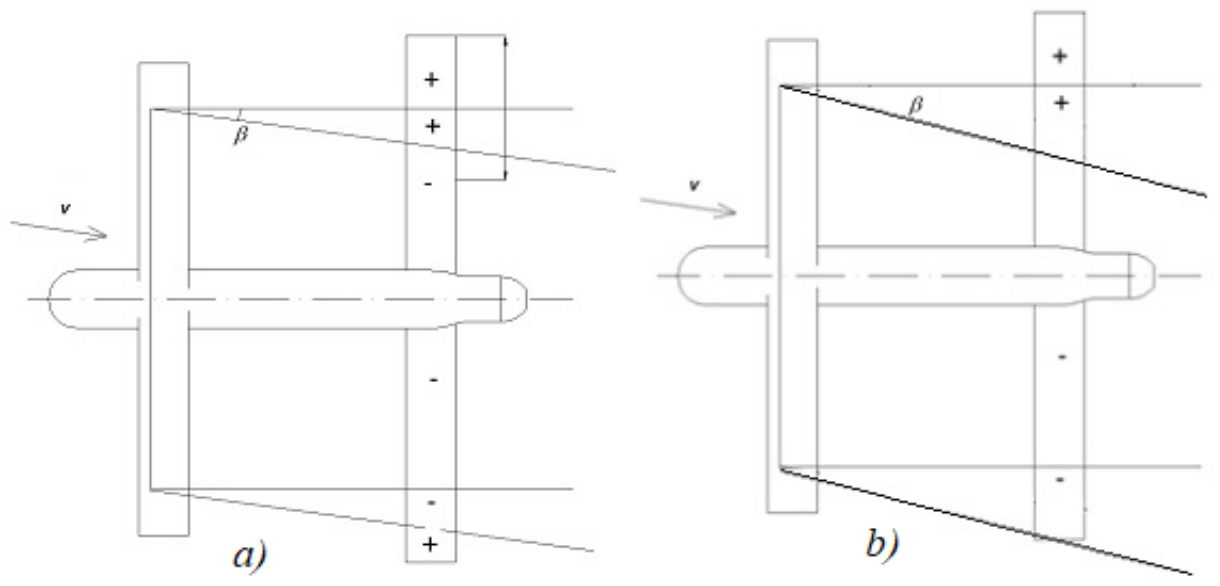

Fig. 9. Wing-wing aerodynamic interference at non-zero sideslip angle

Of course the analytical method considers only linear tip vortices and simply geometrically a forward vortex crosses a tip section of the back wing at $\beta=5 \ldots 10^{\circ}$. Unfortunately, visualization in the wind tunnel was not performed for $\beta>5^{\circ}$, so we cannot directly compare the vortex positions at high sideslip angles. Nevertheless, quantitatively experiment confirmed analytical predictions of roll moment with high accuracy. So the only possible cause of the kink on the roll graph is the forward vortex shifting outside the back wings 'span. Meanwhile, the CFD method indicates that both tip vortices of the forward wings for different geometrical parameters lay between vortices of the back wings at least up to $\beta=20^{\circ}$ (fig. 10). So naturally, there is no kink in CFD dependence of roll moment coefficient but rather smooth changing of derivative (fig. 11).
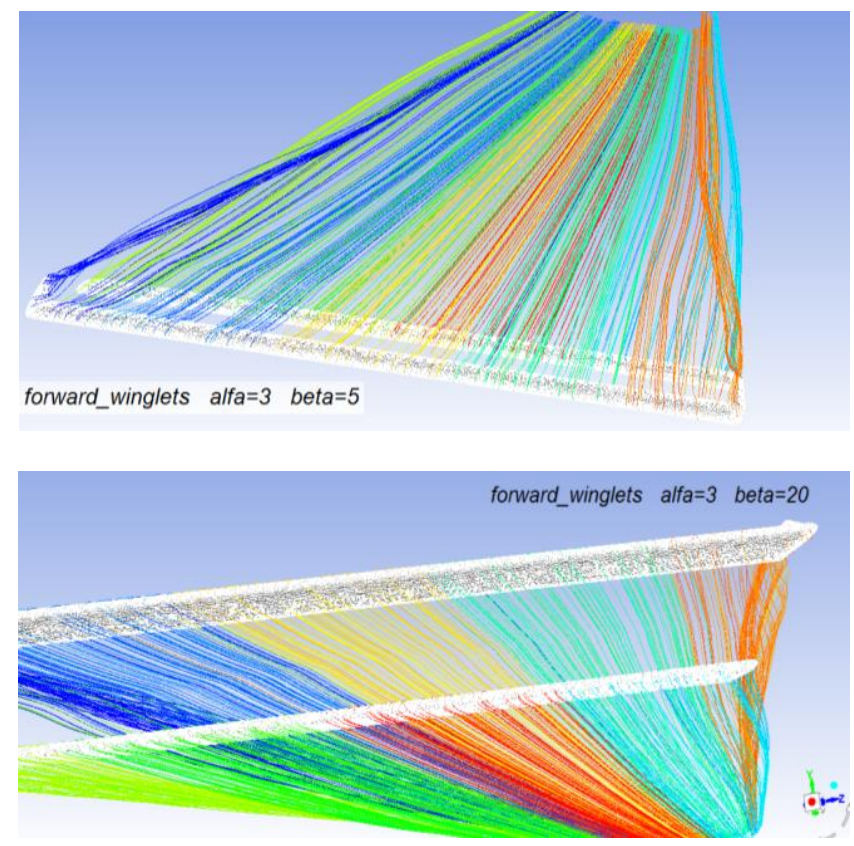

Fig. 10. Pathlines of forward and back wings (winglets on the forward wings) 


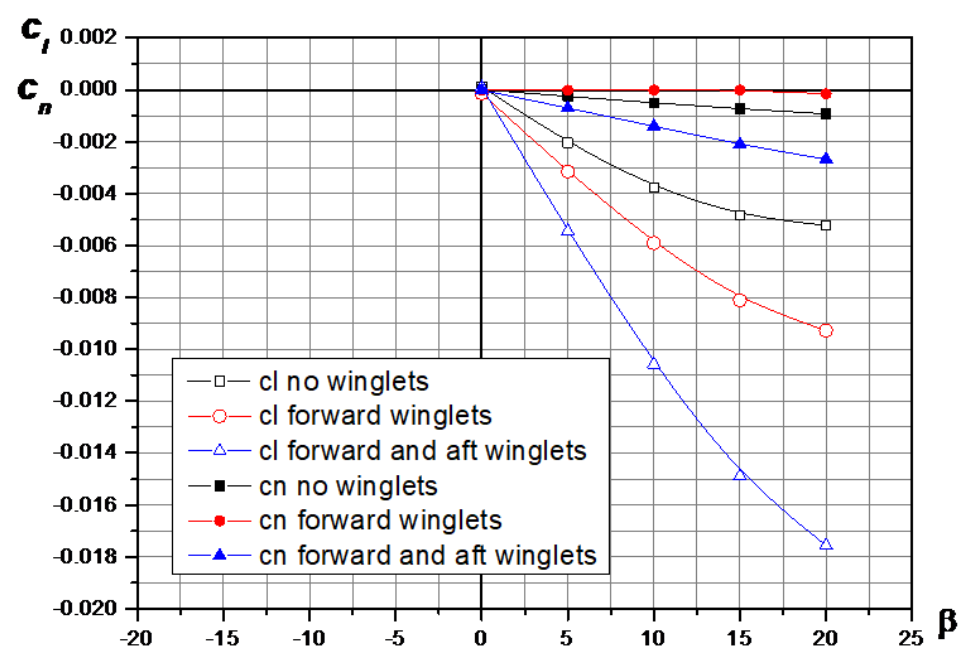

Fig. 11. Roll and yaw moment coefficients according to the CFD calculation

\section{Yaw static moment}

Yaw static moment depends on wings' dihedral angles stronger than for conventional scheme. As wings are far from the center of gravity so dihedral angles of forward/back wings create strong destabilizing/stabilizing yaw moment (fig. 12). So winglets on forward wings decrease directional stability as well, but on back wings increase it greatly.

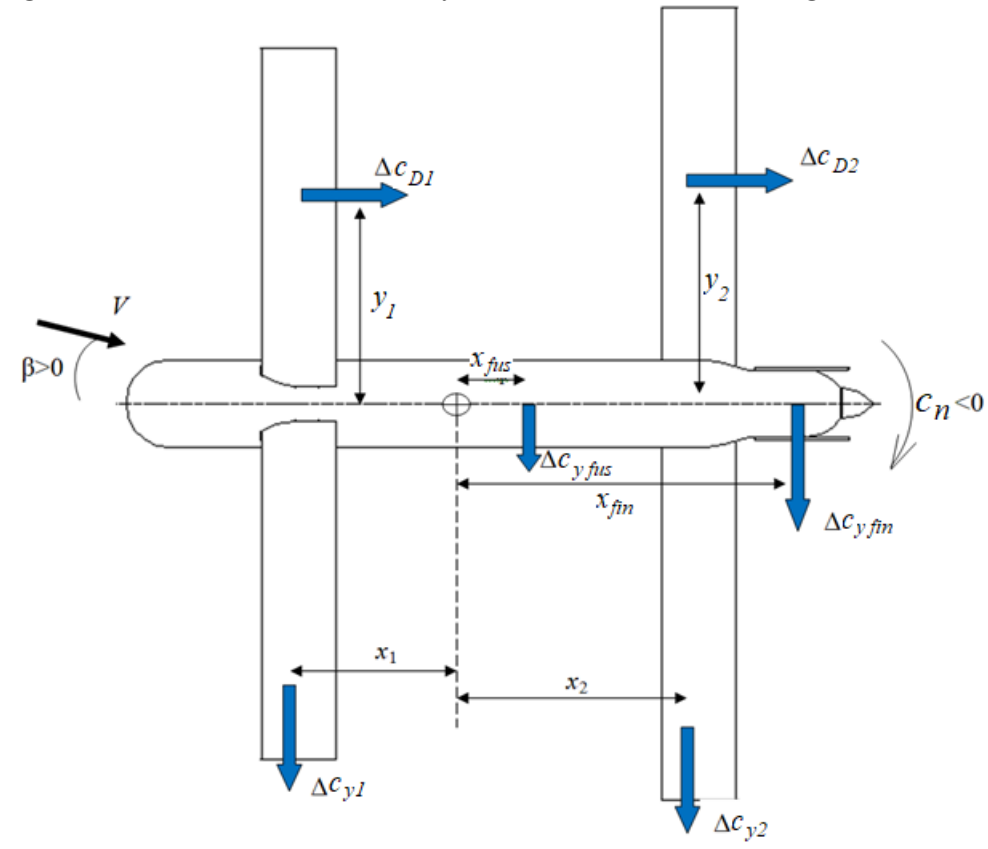

Fig. 12. Yaw moment scheme

For monoplane, the yaw moment is produced mainly by fin and fuselage, so it can be written as 


$$
c_{n I}^{\beta}=c_{n \text { fus } I}^{\beta}+c_{n \text { fin } I}^{\beta}=-c_{y \text { fus }}^{\beta} \frac{S_{M}}{S_{1}} \frac{x_{\text {fus }}}{l_{1}}-c_{y \text { fin }}^{\beta} \frac{S_{\text {fin }}}{S_{1}} \frac{L_{\text {fin }}}{l_{1}} .
$$

For tandem-scheme aircraft, we take into account forward and back wings as well:

$$
\begin{aligned}
& c_{n I I}^{\beta}=c_{n \text { wing } 1}^{\beta}+c_{n \text { wings } 2}^{\beta}+c_{n \text { fus }}^{\beta}+c_{n \text { fin }}^{\beta}=c_{y 1}^{\beta} \beta \frac{S_{1} t g\left|\psi_{1}\right|}{S_{1}+S_{2}} \frac{2 x_{1}}{l_{1}+l_{2}}- \\
& -c_{y 2}^{\beta} \beta \frac{S_{2} t g\left|\psi_{2}\right|}{S_{1}+S_{2}} \frac{2 x_{2}}{l_{1}+l_{2}}-c_{y \text { fus }}^{\beta} \frac{S_{M}}{S_{1}+S_{2}} \frac{2 x_{\text {fus }}}{l_{1}+l_{2}}-c_{y \text { fin }}^{\beta} \frac{S_{\text {fin }}}{S_{1}+S_{2}} \frac{2 L_{\text {fin }}}{l_{1}+l_{2}},
\end{aligned}
$$

where $\psi_{1}$ and $\psi_{2}$ are forward and back wings ' dihedral angles, respectively;

$c_{y 1}^{\beta}$ and $c_{y 2}^{\beta}$ can be estimated as for twin-fin tail with the area of projection on symmetry plane equaled $S_{1} \operatorname{tg}\left|\psi_{1}\right|$ and $S_{2} \operatorname{tg}\left|\psi_{2}\right|$, respectively. According to the well-known method for one fin of low aspect ratio [9], this value can be approximated as

$$
c_{y 1}^{\beta}=0.010+0.011 A R_{\text {vert } 1} \text {, }
$$

where we assume that

$$
A R_{\text {vert } 1}=l_{1} \operatorname{tg}\left|\psi_{1}\right| / b_{1} \text {. }
$$

So for the wings with mean chords $b_{1}$ and $b_{2}$ we can write as for twin-fin tail:

$$
c_{y 1}^{\beta}=0.010+0.011 l_{1} \operatorname{tg}\left|\psi_{1}\right| / b_{1} \text { and } c_{y 2}^{\beta}=0.010+0.011 l_{2} \operatorname{tg}\left|\psi_{2}\right| / b_{2} .
$$

Directional stability degrees predicted by the analytical method (from known monoplane characteristics) and in wind tunnel tests in the range of sideslip angles from $-15^{\circ}$ to $+15^{\circ}$ are presented in table 1 and fig. 13 .

Table 1. Values of $\mathrm{c}_{\mathrm{n}}{ }^{\beta}, 10^{-3}$ of the tandem-scheme model (configuration with all short wings)

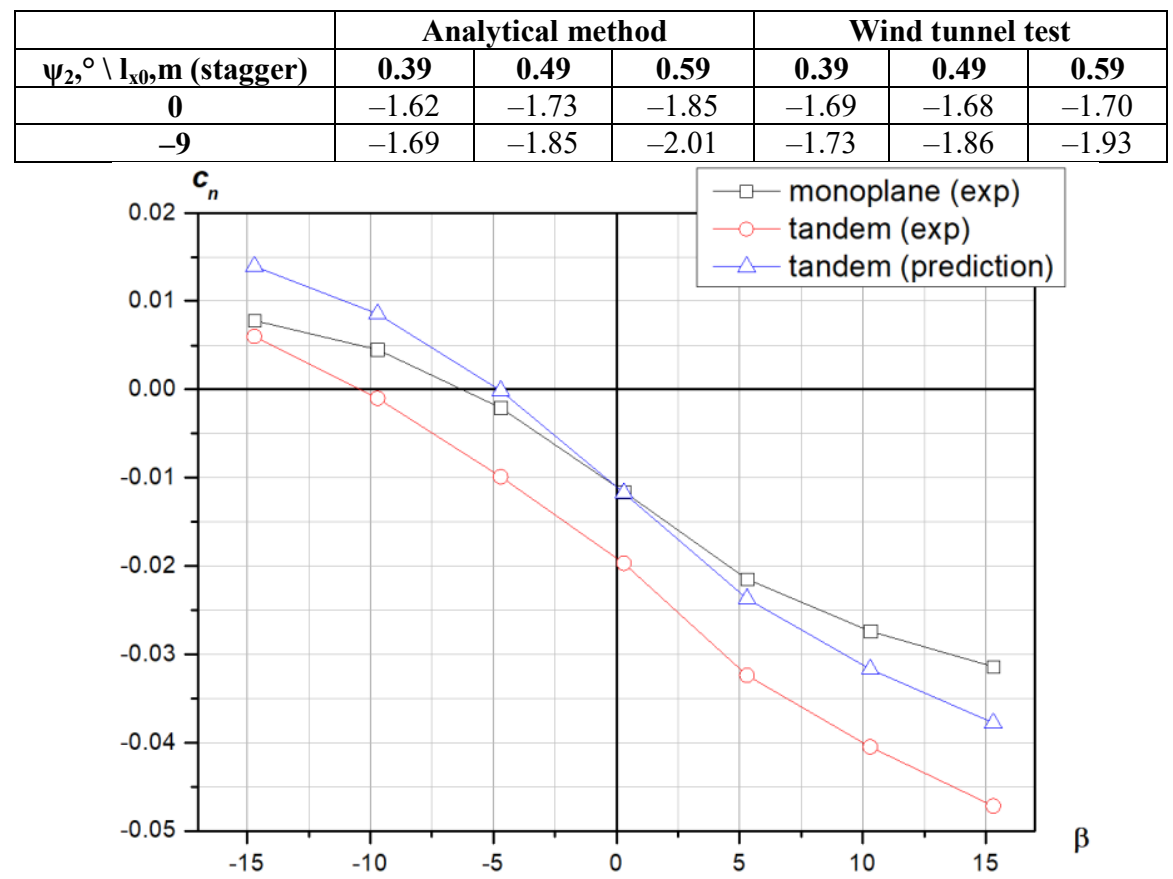

Fig. 13. Yaw moment coefficient (all short wings, $1_{x 0}=0.49 \mathrm{~m}, \psi_{2}=-9^{\circ}$ ).

Compared to monoplane directional stability of $\mathrm{c}_{\mathrm{n}}{ }^{\beta}=-1.4310^{-3}$ given predictions for tandem $\left(\mathrm{c}_{\mathrm{n}}{ }^{\beta}=(-1.62 \ldots-2.01) \cdot 10^{-3}\right)$ are in quite good agreement with experimental data $\left(\mathrm{c}_{\mathrm{n}}{ }^{\beta}\right.$ $\left.=(-1.69 \ldots-1.93) \cdot 10^{-3}\right)$. It was kept into considerations that real dihedral angles of back 
wings were not $0^{\circ}$ and $-9^{\circ}$, but $+4^{\circ}$ and $-5^{\circ}$ due to bending measured for $\alpha=6.6^{\circ}$ and $\beta=0^{\circ}$. Probably method accuracy can be improved if we consider that different loads for left and right back wings result in different dihedral angles, but such measurements were not performed during the wind tunnel tests.

The interference of forward and back wings doesn't affect yaw stability for a perfectly rigid aircraft (or its model). According to fig. 9, forward wings' tip vortices produce roll moment due to different lift on back wings. Indeed it turns to the different induced drags of left and right back wings. But as for conventional scheme aircraft with wings' dihedral angles, this yaw moment is small (not to confuse with wings' swept angles that provide significant directional stability for values of $20 \mathrm{deg}$ and above). Nevertheless, the wingwing interference can change back wings' bending (e.g. under load the average angle is $\psi_{2}=0^{\circ}$, but $\psi_{2 \text { right }}=+3^{\circ}$ and $\psi_{2 \text { left }}=-3^{\circ}$, and both wings increase yaw stability).

\section{Conclusions}

Aerodynamic interference between forward and back wings significantly affects pitch and roll moments of tandem-scheme aircraft.

In a narrow range of sideslip angles, the interference increases roll stability that was predicted by the analytical method and was proved in the wind tunnel test. Outside this range of sideslip angles, the interference has a low effect on roll moment.

Directional stability is decreased by any dihedral angle (positive or negative) of forward wings and winglets on them but is increased by the same factors for back wings. For a perfectly rigid aircraft (or its model), the aerodynamic interference has a low effect on yaw moment. But for flexible back wings, upwash and downwash changing turn to dihedral angles changing that affect directional stability as well as longitudinal.

The vortex system of tandem-scheme aircraft at the sideslip angle is modeled incorrectly by used CFD method (solving RANS). So while analytical and experimental methods show a good agreement in moment characteristics, further research of CFD methods for tandem-wings aerodynamics is needed (e.g. with structured mesh).

Promising future research of tandem-scheme aerodynamics is to determine flight controls efficiency taking into account the wing-wing interference.

\section{References}

1. Vector Hawk Small Unmanned Aircraft System (sUAS) - https://www.navaltechnology.com/projects/vector-hawk-small-unmanned-aircraft-system-suas/

2. A Solar-Algae Hybrid for an Atlantic Crossing - http://sustainableskies.org/a-solaralgae-hybrid-for-an-atlantic-crossing/

3. Vahana - https://www.airbus-sv.com/projects/1

4. Opener BlackFly - https://en.wikipedia.org/wiki/Opener_BlackFly

5. I. S. Kryvokhatko, V. V. Sukhov, Mechanics of Gyroscopic Systems, 28, 59-71 (2015)

6. I. S. Kryvokhatko, Aerospace Engineering and Technology, 3, 33-40 (2015)

7. O. O. Voitiuk, I. S. Kryvokhatko, Mechanics of Gyroscopic Systems, 34, 98-108 (2017)

8. O. M. Masko, I. S. Kryvokhatko, V. V. Sukhov, Transactions of the Institute of Aviation. Prace Instytutu Lotnictva, 4, 63-75 (2014)

9. Rukovodstvo dlya konstruktorov letatelnyh apparatov samodeyatelnoy postroyki, SIBNIA, 229-231 (1989) 